\title{
Co-Exposure to Multiple Pollutants and Its Cardiovascular Effects in a Subway System — Beijing Municipality, China, 2017
}

\author{
Wenlou Zhang'; Xuan Yang'; Xu Jia ${ }^{1}$; Wei Dong'; Hongyu Li'; Lu Pan ${ }^{1,2}$; Jiao Shan'; \\ Shaowei $\mathrm{Wu}^{3}$; Xinbiao Guo'; Furong Deng ${ }^{1, *}$
}

\begin{abstract}
Summary
What is already known on this topic?

With rapid urbanization, traffic-related air pollution has become a global concern. However, its association with cardiovascular health has not been fully elucidated.

\section{What is added by this report?}

This study provided novel evidence of the joint cardiovascular effect of multiple pollutants in subway cabins, further identified two pollutants that played dominant roles, and validated the effectiveness of targeted interventions.

What are the implications for public health practice?

The findings were helpful to guide the formulation and development of prevention and control strategies for key traffic-related pollutants that endanger the cardiovascular health of commuters.
\end{abstract}

With rapid global urbanization, air and noise pollution in subway systems (also called metro systems) and the potential cardiovascular hazards they pose have become a global public health issue. However, the joint effect of multiple pollutants, as well as the key pollutants that play a dominant role in cardiovascular health, remain unclear. A randomized crossover study with respirator and/or headphone interventions was conducted among healthy young adults from March 11 to May 28, 2017 in the Beijing subway. This study found that co-exposure to size-fractioned particulate matter (PM), black carbon (BC), and noise was strongly associated with changes in heart rate variability (HRV) indices. $\mathrm{BC}$ and noise may be the two dominant pollutants causing the overall impact. Analysis based on data from intervention phases suggested that the impact of $\mathrm{BC}$ or noise might be attenuated or even reversed when reducing the level of another pollutant (noise or $\mathrm{BC}$ ). The findings were helpful in guiding the formulation and development of prevention and control strategies for key traffic-related pollutants that endanger the cardiovascular health of commuters.

Overall, 40 healthy young adults were recruited for this randomized crossover study from March 11 to May 28, 2017, in Beijing. Details of the study implementation could be found in previous publications (1-2). In brief, participants commuted for about 4 hours between 9:00-13:00 in the subway during 4 different periods with/without intervention (wearing respirator and/or headphone). The personal real-time levels of $\mathrm{PM}_{1}$ (aerodynamic diameters < $1 \mu \mathrm{m}$ ), $\mathrm{PM}_{1-2.5}$ (aerodynamic diameter $\geq 1 \mu \mathrm{m}$ and $<$ $2.5 \mu \mathrm{m}$ ), $\mathrm{PM}_{2.5-10}$ (aerodynamic diameter $\geq 2.5 \mu \mathrm{m}$ and $<10 \mu \mathrm{m}), \mathrm{BC}$, and noise were measured. Simultaneously, HRV parameters were obtained using a 12-channel ambulatory ECG monitor. Total power (TP), very-low-frequency power (VLF), low-frequency power (LF), high-frequency power (HF), LF/HF and standard deviation of normal-to-normal intervals (SDNN) were included in this study. Bayesian kernel machine regression (BKMR) was used to further examine the joint health effect of multiple pollutants in the subway cabin. This model allows nonlinear relationships and potential interactions and has been used widely to assess the overall effect of mixed pollutants (3). Posterior inclusion probability (PIP) was estimated through variable selection to assess the relative importance of exposure variables. PIP values range from 0 to 1 and a larger PIP value means a higher importance. All exposure and outcome variables were standardized. The covariates included gender, age, body mass index (BMI), temperature, relative humidity and $\mathrm{CO}_{2}$ level. The number of subjects was used as a random effect term to control the correlation between repeated measurements. The lag effects from $5 \mathrm{~min}$ to 2 hours were examined, and the most significant effect was reported at $30 \mathrm{~min}$ lag. BKMR analysis was conducted using $\mathrm{R}$ software (version 4.0.3; $\mathrm{R}$ project for Statistical Computing) with the "bkmr" package. The study protocol was approved by the Institutional Review Board of Peking University Health Science 
Center (IRB number: 00001052-16066) and informed consent was obtained from each participant.

In total, 39 participants completed this study, of whom $18(46.2 \%)$ were females. Their mean age and BMI were 21.2 years and $21.6 \mathrm{~kg} / \mathrm{m}^{2}$, respectively. The mean levels of $\mathrm{PM}_{1}, \mathrm{PM}_{1-2.5}, \mathrm{PM}_{2.5-10}, \mathrm{BC}$, and noise were $34.1,51.6,145.2,9.5 \mu \mathrm{g} / \mathrm{m}^{3}$, and $75.9 \mathrm{dBA}$, respectively. Increased levels of co-exposure to these pollutants were significantly associated with decreased TP, VLF, SDNN, and increased LF/HF (Figure 1). According to the results of PIPs in Table 1, BC had a higher PIP than other pollutants in most HRV indices, ranging from 0.69 to 1.00 . Specifically, $\mathrm{BC}$ had the highest PIP value in TP (1.00), VLF (1.00), LF (1.00), and SDNN (0.96), indicating the largest contribution to the overall effect. Noise was the second most important because it had a high PIP in LF (1.00), HF (0.96), and LF/HF (0.72).

To clarify the effectiveness and necessity of $\mathrm{BC}$ and noise prevention and control in the subway system, the exposure-response relationships of $\mathrm{BC}$ and noise with $\mathrm{HRV}$ indices were plotted based on the data from both no-intervention and headphone/respirator intervention phases. As shown in Figure 2, a weaker effect of $\mathrm{BC}$ on VLF, LF, HF, and SDNN was observed in the headphone intervention phase (low noise level) than the no-intervention one (high noise level), and the effect on LF/HF was even slightly reversed from a positive to negative association. For the effect of noise on all HRV indices, the relationships significantly differed in the respirator intervention phase (low BC concentration), and positive associations between noise
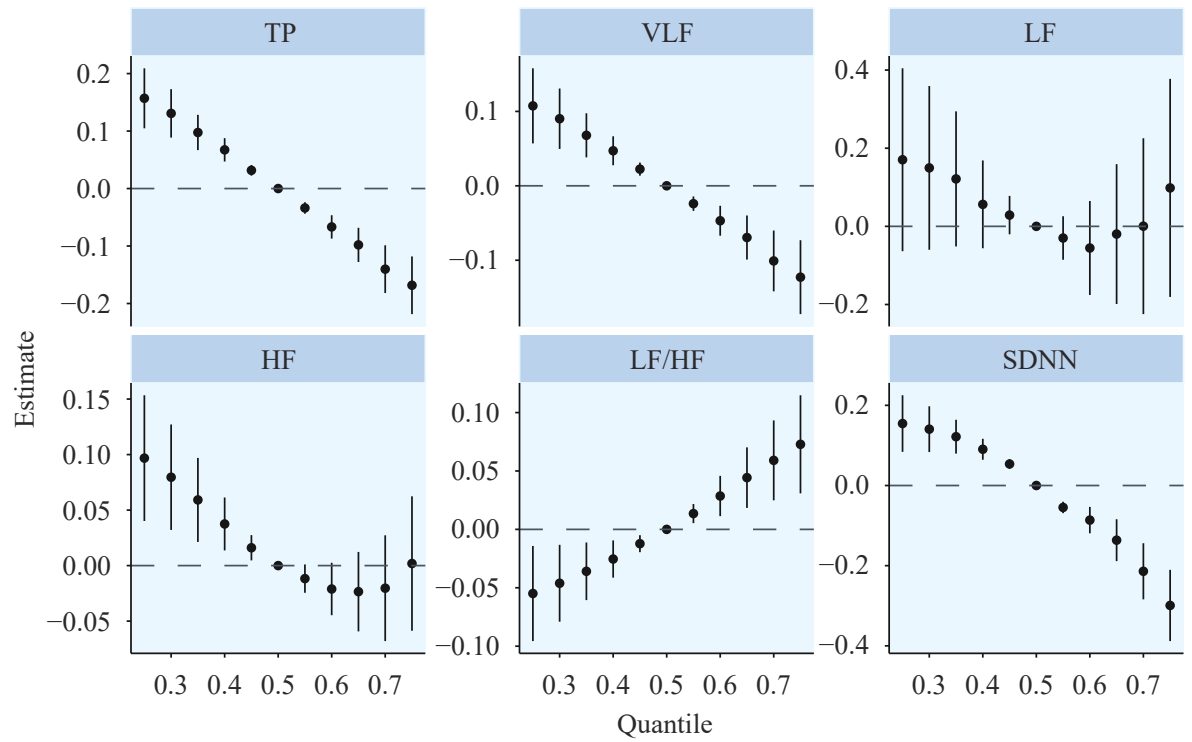

FIGURE 1. The joint effect estimates and 95\% confidence interval $(\mathrm{Cl})$ of multiple air pollutants $\left(\mathrm{PM}_{1}, \mathrm{PM}_{1-2.5}, \mathrm{PM}_{2.5-10}, \mathrm{BC}\right.$, and noise) in subway cabin on HRV parameters of study participants in Beijing, 2017

Note: The plot compared each HRV index when all exposures were at a particular quantile to when all were at the median (reference).

Abbreviations: TP=total power; VLF=very low frequency power; LF=low frequency power; HF=high frequency power; $\mathrm{SDNN}=$ standard deviation of normal-to-normal intervals; HRV=heart rate variability.

TABLE 1. Posterior inclusion probabilities (PIPs) from basyesian kernel machine regression model for heart rate variability parameters of healthy young adults in Beijing, $2017^{*}$.

\begin{tabular}{ccccccc}
\hline Variable & TP & VLF & LF & HF & LF/HF & SDNN \\
\hline $\mathrm{PM}_{1}$ & 0.51 & 0.45 & 1.00 & 0.80 & 0.61 & 0.26 \\
$\mathrm{PM}_{1-2.5}$ & 0.48 & 0.43 & 0.76 & 0.70 & 0.73 & 0.27 \\
$\mathrm{PM}_{2.5-10}$ & 0.59 & 0.63 & 1.00 & 0.75 & 0.76 & 0.62 \\
Black carbon & 1.00 & 1.00 & 1.00 & 0.90 & 0.69 & 0.96 \\
Noise & 0.60 & 0.53 & 1.00 & 0.96 & 0.72 & 0.21 \\
\hline
\end{tabular}

Abbreviations: $\mathrm{PM}=$ particulate matter; TP=total power; $\mathrm{VLF}=$ very low frequency power; $\mathrm{LF}=$ low frequency power; HF=high frequency power; SDNN=standard deviation of normal-to-normal intervals.

* PIP is a measure of the importance of exposure variables, and a larger value means a higher importance. 
(A) $\mathrm{BC}$
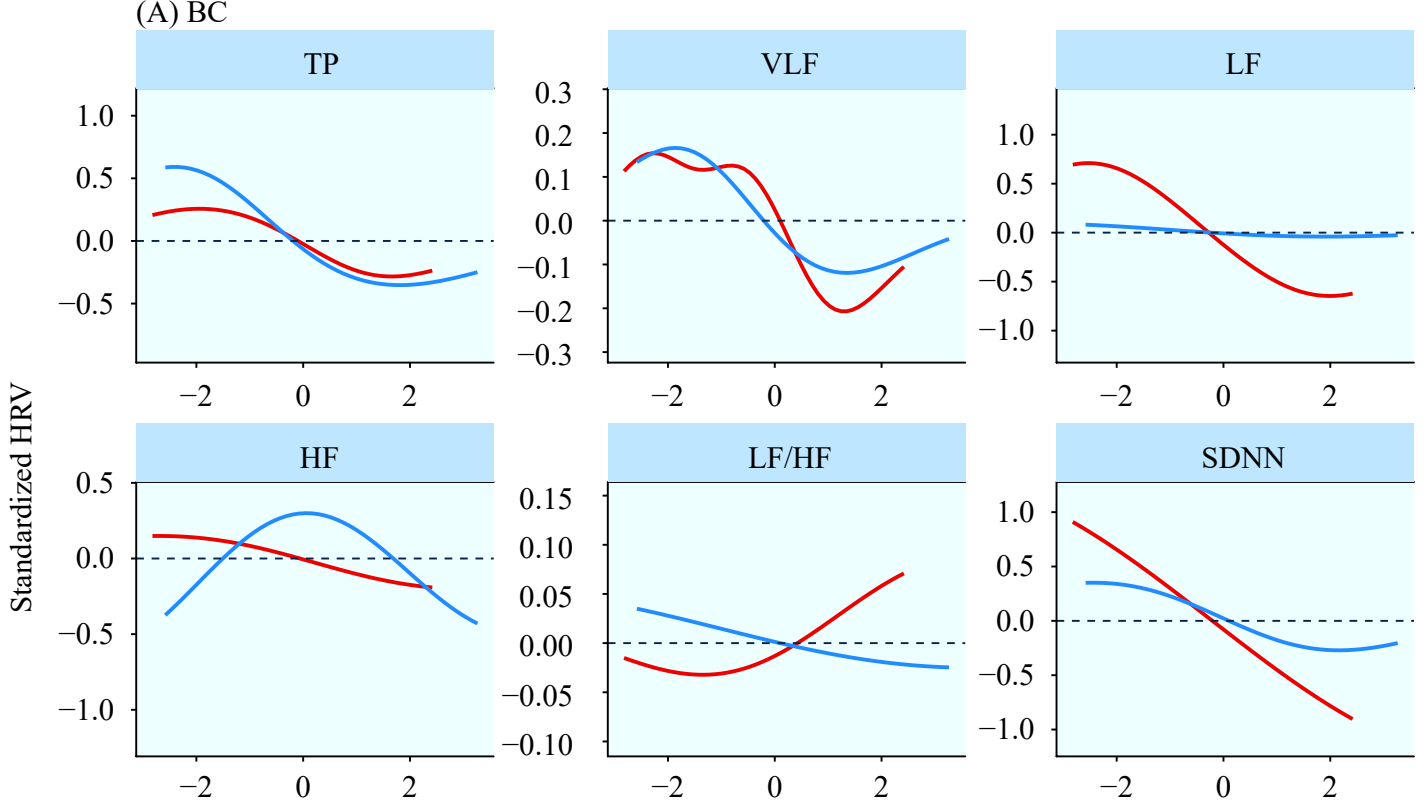

Standardized BC

— No intervention - Headphone intervention

(B) Noise

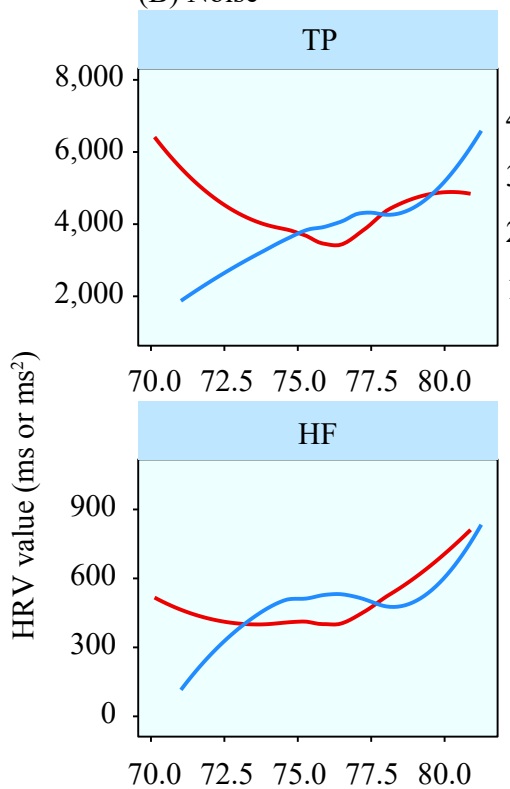

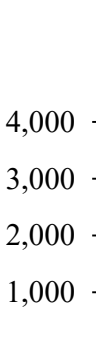

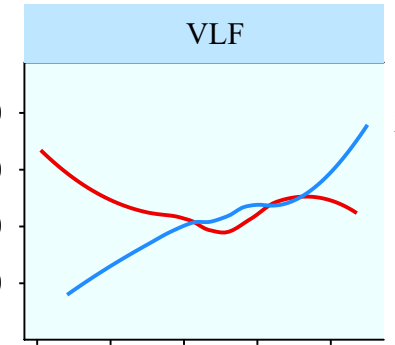

$\begin{array}{lllll}70.0 & 72.5 & 75.0 & 77.5 & 80.0\end{array}$

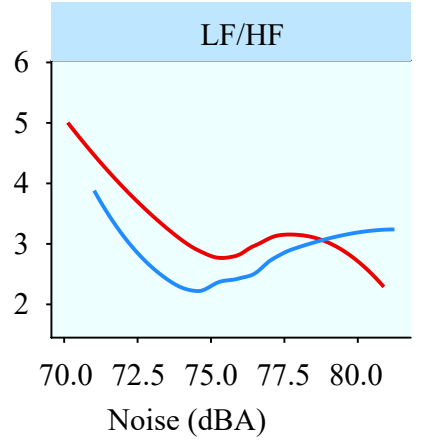

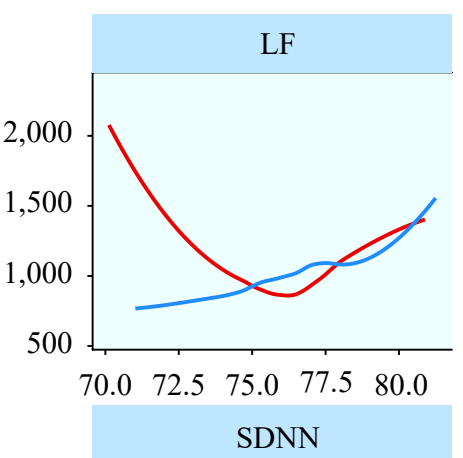

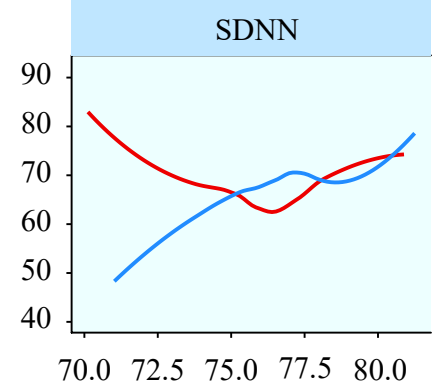

— No intervention

— Respirator intervention

FIGURE 2. The exposure-response relationship of black carbon $(\mathrm{BC})$ and noise in subway cabin with heart rate variability (HRV) indices of participants in Beijing, 2017.

(A) Comparison of the effects of BC on HRV indices between no intervention (high-noise level) and headphone intervention (low-noise level) phase based on basyesian kernel machine regression analysis; (B) Comparison of the effects of noise on HRV indices between no intervention (high-BC concentration) and headphone intervention (low-BC concentration) phase based on LOESS.

Abbreviations: $\mathrm{BC}=$ black carbon; $\mathrm{HRV}=$ heart rate variability; $\mathrm{TP}=$ total power; $\mathrm{VLF}=\mathrm{very}$ low frequency power; $\mathrm{LF}=\mathrm{low}$ frequency power; HF=high frequency power; SDNN=standard deviation of normal-to-normal intervals; LOESS=locally weighted regression. 
and TP, VLF, LF, HF, and SDNN were found during this phase.

\section{DISCUSSION}

The metro system has become an indispensable part of megacities, and its environmental quality and potential health hazards are attracting more and more attention. This study found that short-term $(30 \mathrm{~min})$ co-exposure to size-fractioned PM, BC, and noise was strongly associated with changes in HRV indices. HRV can reflect the activities of the sympathetic nervous system (SNS) and the parasympathetic nervous systems (PNS), and its reduction has been related to higher cardiovascular risks (4). TP and SDNN are global indicators of HRV, and VLF is thought to be strongly associated with all-cause mortality and other diseases. LF/HF was thought to represent the balance of SNS and PNS and is positively related to SNS activity. In this study, positive association with LF/HF was found, indicating SNS activity increased more than PNS, which was consistent with previous studies (2-3,5-6).

To achieve the maximum input-output ratio of pollution prevention and control based on health benefits, it is imperative to identify the pollutants that contribute most to health hazards. The study found that $\mathrm{BC}$ mediated most of the cardiovascular health damage, followed by noise. The effects of $\mathrm{BC}$ and noise on cardiac autonomic nerve function have also been demonstrated in other studies (5-7). BC is mainly emitted by traffic transport, where a high level of noise is usually produced simultaneously. They may be two crucial targets for traffic-related air pollution prevention and control, and they seem to deserve more concern in the subway system because of the higher exposure levels compared to other modes of transport, including taxi, bus, cycling, and walking (8-9).

Our previous study demonstrated the cardiovascular benefits of respirator and headphone interventions (2). Considering the potential interaction of $\mathrm{BC}$ and noise on cardiovascular health (6-7), this study further explored the exposure-response relationships between $\mathrm{BC}$ or noise and HRV indices, controlling each pollutant at different levels. When reducing the exposure level of $\mathrm{BC}$ or noise using respirators or headphones, the adverse effects of another pollutant (noise or BC) on cardiac autonomic nerve function were significantly attenuated and even reversed. The results further validated the effectiveness of the target intervention. In addition, the findings also suggested that healthy adults may develop a protective compensatory response to the adverse effects of single pollutant (BC or noise), though it may be not enough when co-exposed to multiple pollutants. Similarly, a panel study found a weaker association between BC and HRV indices when noise was controlled in the regression model (G). Another randomized crossover study also observed the effect of BC on HRV indices of health adults was stronger in a high noise environment (traffic center) than a low noise one (park) ( 7 ).

This is the first study to explore the joint effect of multiple pollutants in the subway on the cardiac autonomic function and to determine which pollutants deserve particular concern in a near-real exposure scenario. This study further clarified the effectiveness and necessity of targeted interventions for air pollution in the subway system.

This study was still subject to some limitations. First, gaseous pollutants were not included. Second, participants in this study were healthy young adults, which might limit the generalizability of the findings. But it could be expected that similar or even stronger effects might be observed in susceptible populations.

In summary, short-term co-exposure to multiple pollutants could disturb the cardiac autonomic function. BC and noise may be the two pollutants with the greatest contribution. In addition to the direct cardiovascular benefits of wearing a respirators or headphones (2), this study further verified that such interventions were also helpful to reduce the susceptibility of the cardiovascular system to other pollutants, possibly because the compensatory response of healthy young people is adequate to cope with shortterm high levels of exposure to a single pollutant. Therefore, this study confirmed the potential public health implications of traffic-related air pollution interventions, and highlighted that $\mathrm{BC}$ and noise may be key to urban traffic-related air pollution prevention and control to avoid cardiovascular lesions. It is urgent and necessary for individuals and relevant departments to take targeted measures to protect cardiovascular health from air pollution during commuting.

Funding: The National Key Research and Development Program of China (2016YFC0206506, 2017YFC0702701).

\section{doi: $10.46234 / \mathrm{ccdcw} 2021.233$}

\# Corresponding author: Furong Deng, lotus321321@126.com.

\footnotetext{
${ }^{1}$ Department of Occupational and Environmental Health Sciences, School of Public Health, Peking University, Beijing, China; ${ }^{2}$ Qingdao Municipal Center for Disease Control and Prevention, Qingdao Institute of Preventive Medicine, Qingdao, Shandong, China; ${ }^{3}$ Department of Occupational and Environmental Health Sciences,
} 
School of Public Health, Xi'an Jiaotong University Health Science Center, Xi'an, Shaanxi, China.

Submitted: October 23, 2021; Accepted: November 02, 2021

\section{REFERENCES}

1. Yang X, Jia X, Dong W, Wu S, Miller MR, Hu D, et al. Cardiovascular benefits of reducing personal exposure to traffic-related noise and particulate air pollution: a randomized crossover study in the Beijing subway system. Indoor Air 2018;28(5):777 - 86. http://dx.doi.org/10. 1111 /ina. 12485 .

2. Jia X, Yang X, Hu DY, Dong W, Yang F, Liu Q, et al. Short-term effects of particulate matter in metro cabin on heart rate variability in young healthy adults: impacts of particle size and source. Environ Res 2018;167:292 - 8. http://dx.doi.org/10.1016/j.envres.2018.07.017.

3. Bobb JF, Valeri L, Henn BC, Christiani DC, Wright RO, Mazumdar M, et al. Bayesian kernel machine regression for estimating the health effects of multi-pollutant mixtures. Biostatistics 2015;16(3):493 - 508. http:// dx.doi.org/10.1093/biostatistics/kxu058.

4. Shaffer F, Ginsberg JP. An overview of heart rate variability metrics and norms. Front Public Health 2017;5:258. http://dx.doi.org/10.3389/ fpubh.2017.00258.

5. Pan L, Wu SW, Li HY, Xu JH, Dong W, Shan J, et al. The short-term effects of indoor size-fractioned particulate matter and black carbon on cardiac autonomic function in COPD patients. Environ Int 2018; 112:261 - 8. http://dx.doi.org/10.1016/j.envint.2017.12.037.

6. Biel R, Danieli C, Shekarrizfard M, Minet L, Abrahamowicz M, Baumgartner $\mathrm{J}$, et al. Acute cardiovascular health effects in a panel study of personal exposure to traffic-related air pollutants and noise in Toronto, Canada. Sci Rep 2020;10(1):16703. http://dx.doi.org/10. 1038/s41598-020-73412-6.

7. Huang J, Deng FR, Wu SW, Lu H, Hao Y, Guo XB. The impacts of short-term exposure to noise and traffic-related air pollution on heart rate variability in young healthy adults. J Expo Sci Environ Epidemiol 2013;23(5):559 - 64. http://dx.doi.org/10.1038/jes.2013.21.

8. Li B, Lei XN, Xiu GL, Gao CY, Gao S, Qian NS. Personal exposure to black carbon during commuting in peak and off-peak hours in Shanghai. Sci Total Environ 2015;524-525:237 - 45. http://dx.doi.org/10.1016/j. scitotenv.2015.03.088.

9. Liu YS, Lan BW, Shirai J, Austin E, Yang CH, Seto E. Exposures to air pollution and noise from multi-modal commuting in a Chinese city. Int J Environ Res Public Health 2019;16(14):2539. http://dx.doi.org/10. 3390/ijerph16142539. 